Supporting Information

\title{
Fabry-Pérot Phonon Polaritons in Boron Nitride Nanotube Resonators
}

Cassandra Phillips, Yi-Fang Lai, Gilbert C. Walker*

Department of Chemistry, University of Toronto, 80 St. George Street, Toronto, Ontario M5S

3H6, Canada

Corresponding Author

*E-mail: gilbert.walker@utoronto.ca 


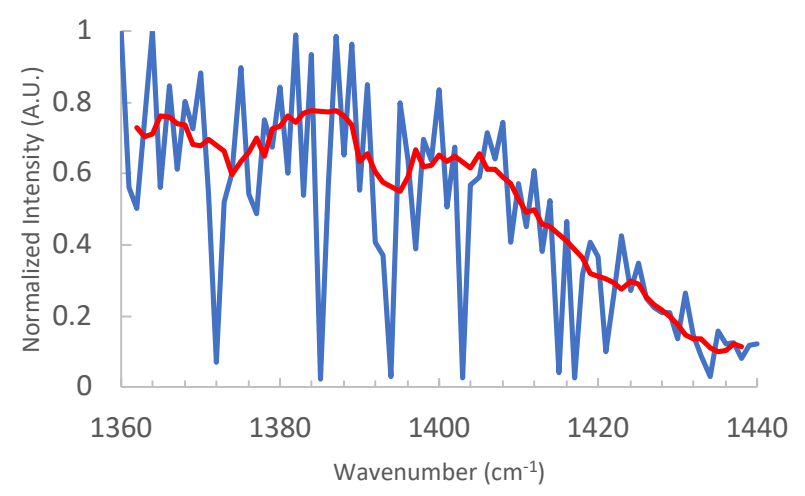

Figure S1. Quantum cascade laser power spectrum (blue) and five-point smoothed power spectrum (red). Laser power was normalized to the intensity at $1360 \mathrm{~cm}^{-1}$. Smoothening process excluded outlier data points $\left(1372,1385,1394,1403,1415,1417 \mathrm{~cm}^{-1}\right)$. The smoothened spectrum was used for the Fabry-Pérot model as only the broad trends in laser power variation were of interest.

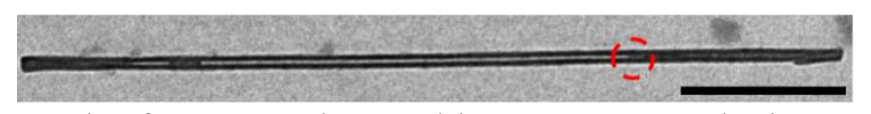

Figure S2. TEM micrograph of $2.5 \mu \mathrm{m}$ tube used in s-SNOM analysis. Red circle around internal barrier on the end of the tube seen as the top region of the spatiospectral image. Scale bar is 500 nm.
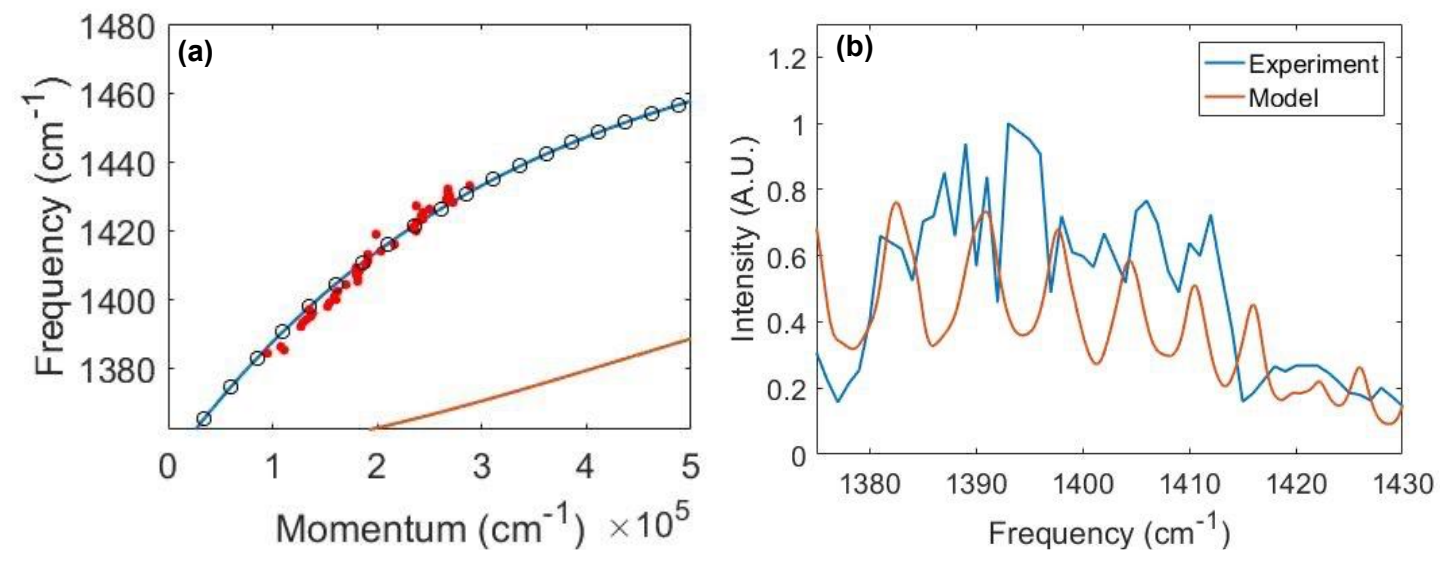
Figure S3. (a) Dispersion relationship for the $2.5 \mu \mathrm{m}$ BNNT. Experimental data, marked with red circles, follows closely with the $1=0$ mode of the HPhP dispersion relationship (blue curve). Fabry-Pérot resonances values are marked with open black circles, there is no correlation between the resonances and experimental momenta as expected for a non-resonant tube. (b) Peak-to-peak fringe amplitude for $2.5 \mu \mathrm{m}$ tube compared to modeled Fabry-Pérot amplitude. As expected, there is no correlation between experimental and modeled data.

\section{Phase-shift for the tube ends of the $2.5 \mu \mathrm{m}$ BNNT}

Using a similar treatment to quantify the reflection off single terminals when Fabry-Perot resonances are not supported in the entire tube, as in the case of the $2.5 \mu \mathrm{m}$ long tube, the equation describing the constructive reflection from a single tube end becomes

$$
2 k_{P H P} l_{\text {fringe }}+\phi_{R}=n \pi
$$

Where $1_{\text {fringe }}$ is the distance between the first fringe and the closest end of the tube. This is related to the observed PhP standing wave within the longer tube. For the $2.5 \mu \mathrm{m}$ tube, a phase-shift value of approximately $2.2 \pm 0.1$ and $2.1 \pm 0.2$ radians are found for the left and the right-side terminals, respectively. This result is notably different from the values seen for the short, resonant tube (Fig. 3). The non-resonant phase shows a phase-change that is largely independent of wavevector due to the absence of resonant modes. BNNTs grown through CVD have a large degree of morphological variation, including having open or closed terminals. Given that the measured standing wave reflects off the terminals before being detected, these variations, including open or closed ends and tapering near the ends, would be expected to have a notable effect on the reflected phase by modifying the quality of the dielectric interface. Both ends of the 
$2.5 \mu \mathrm{m}$ tube show similar characteristics, including closed terminals and no measurable tapering (see Supporting Figure 2), and have a statistically similar resulting phase gain.

\section{Analytical and Computational Methods}

The BNNT permittivities $\varepsilon_{\|}$and $\varepsilon_{\perp}$ were described using the Lorentz model below.

$$
\varepsilon_{\mu}=\varepsilon_{\infty, \mu}\left(1+\frac{\omega_{L O, \mu}^{2}-\omega_{T O, \mu}^{2}}{\omega_{T O, \mu}^{2}-\omega^{2}-i \omega \Gamma_{\mu}}\right), \mu=\perp, \|
$$

Where $\varepsilon_{\infty}$ is the high-frequency permittivity, $\omega_{T O}$ and $\omega_{L O}$ are the TO and LO phonon

frequencies, respectively, and $\Gamma$ is the damping constant. Values for the high-frequency permittivities are taken from analysis into the normal modes of $\mathrm{h}-\mathrm{BN} .{ }^{1}$ For the dielectric function perpendicular to the optical axis, the TO frequency is taken to be $1355 \mathrm{~cm}^{-1}$, and the LO is associated with the $\varepsilon=0$ crossing at $1527 \mathrm{~cm}^{-1}$, respectively. Experimental far-field IR values for the TO mode of BNNTs show a large degree of variability from $1377 \mathrm{~cm}^{-1}$ to $1317 \mathrm{~cm}^{-1}$ based on tube quality and purification, the value of $1355 \mathrm{~cm}^{-1}$ was chosen to best fit the data using the dispersion relationship. ${ }^{2,3}$ The damping constant $\Gamma$ was chosen to be $8 \mathrm{~cm}^{-1}$ to match observed decay lengths in experimental data. The values for the dielectric function parallel to the optical axis as well as the high-frequency permittivities were taken from the analysis of h-BN.

The phonon polariton dispersion is described using a hyperbolic phonon polariton dispersion modified using an effective medium approximation: ${ }^{4}$

$$
\begin{gathered}
q(\omega)+i \kappa(\omega)=-\frac{\psi}{d}\left[\arctan \left(\frac{\varepsilon_{a i r}}{\varepsilon_{\perp, e f f} \psi}\right)+\arctan \left(\frac{\varepsilon_{S}}{\varepsilon_{\perp, e f f} \psi}\right)+\pi \ell\right], \psi=\frac{\sqrt{\varepsilon_{\|}}}{i \sqrt{\varepsilon_{\perp}}}(6) \\
\varepsilon_{e f f}=\frac{\varepsilon_{\mathrm{m}}\left(2 \delta_{i}\left(\varepsilon_{i}-\varepsilon_{m}\right)+\varepsilon_{i}+2 \varepsilon_{m}\right)}{2 \varepsilon_{m}+\varepsilon_{i}-\delta_{i}\left(\varepsilon_{i}-\varepsilon_{m}\right)}(7)
\end{gathered}
$$


The modifications to the model are primarily described here. ${ }^{5} \mathrm{q}$ and $\kappa$ are the real and imaginary portions of the dispersion relationship respectively, $\varepsilon_{\text {air }}$ and $\varepsilon_{s}$ are the dielectric contributions from the air and substrate, and $\ell$ is the integer mode number corresponding to the branch of the hyperbolic dispersion. The thicknesses of the BNNTs, $d$, were taken to be $16 \mathrm{~nm}$ and $38 \mathrm{~nm}$ for the $0.88 \mu \mathrm{m}$ and $2.5 \mu \mathrm{m}$ tubes, respectively. To obtain the final effective values for the dielectric function, the values of $\varepsilon_{\|}$and $\varepsilon_{\perp}$ from eqn. 5 were inserted into the Maxwell-Garnett approximation (eqn. 7). The effective medium approximation has a variable fraction for describing the air-channel contribution, $\delta_{i}$. The effective medium dielectric functions were combined in the analytical dispersion relationship (eqn. 6) to produce the final dispersion simulation.

Finite element method simulations were performed using the commercial software package COMSOL. Calculations on the scattered field were performed on a 2D cut along a cylindrical structure with a platinum sphere included to model the behavior of the AFM probe. The values of $\varepsilon^{\infty}, \omega_{T O}, \omega_{L O}, \Gamma, \varepsilon_{a i r}, \varepsilon_{s}$, and $\mathrm{d}$ used were the same as the parameters above in MATLAB, with no consideration given to the effective medium approximation. For the scattered field for h-BN, identical parameters were used from previous studies on phonon polaritons bwithin h-BN. ${ }^{4}$

\section{Differences between height and widths observed between AFM and TEM}

We examined tubes with both AFM/s-SNOM imaging as well as TEM imaging. We report a AFM thicknesses of $16 \mathrm{~nm}$ and $38 \mathrm{~nm}$ respectively for the $0.88 \mu \mathrm{m}$ and $2.5 \mu \mathrm{m}$ tubes and widths of $40 \mathrm{~nm}$ for both tubes through TEM. AFM measurements were done in tapping mode with the same probe and set-point. Through TEM we also report an inner diameter of $20 \mathrm{~nm}$ and $16 \mathrm{~nm}$ 
respectively for the inner diameters of the $0.88 \mu \mathrm{m}$ and $2.5 \mu \mathrm{m}$ tubes. However, electron contrast in TEM likely overestimates the thickness of the walls due to the changing scattering crosssection with changing orientation of the tube wall. We suggest that the number of inner walls has an effect on the wetting and deformability of tubes with the 0.88 um tube deforming more to wet the surface. ${ }^{6}$

Nevertheless, the treatment in both the analytical and computational models used to compare the experimental results is agnostic to variations in tube morphology. In the analytical model we only consider the volume fraction of the air channel within the entire sampled volume which, due to the comparatively large probe radius $(\sim 20 \mathrm{~nm})$, represents the entire tube cross section. In the COMSOL FEM simulation, only a 2D cross-section along the length of the tube is considered without any treatment for the overall morphology.

\section{References}

(1) Geick, R.; Perry, C. H.; Rupprecht, G. Normal Modes in Hexagonal Boron Nitride. Phys. Rev. 1966, 146 (2), 543-547. https://doi.org/10.1103/PhysRev.146.543.

(2) Borowiak-Palen, E.; Pichler, T.; Fuentes, G. G.; Bendjemil, B.; Liu, X.; Graff, A.; Behr, G.; Kalenczuk, R. J.; Knupfer, M.; Fink, J. Infrared Response of Multiwalled Boron Nitride Nanotubes. Chem. Commun. 2003, 0 (1), 82-83. https://doi.org/10.1039/B208214D.

(3) Amin, M. S.; Molin, T. E.; Tampubolon, C.; Kranbuehl, D. E.; Schniepp, H. C. Boron Nitride Nanotube Impurity Detection and Purity Verification. Chem. Mater. 2020, 32 (21), 9090-9097. https://doi.org/10.1021/acs.chemmater.0c03609.

(4) Dai, S.; Fei, Z.; Ma, Q.; Rodin, A. S.; Wagner, M.; McLeod, A. S.; Liu, M. K.; Gannett, W.; Regan, W.; Watanabe, K.; Taniguchi, T.; Thiemens, M.; Dominguez, G.; Neto, A. H. C.; Zettl, A.; Keilmann, F.; Jarillo-Herrero, P.; Fogler, M. M.; Basov, D. N. Tunable Phonon Polaritons in Atomically Thin van Der Waals Crystals of Boron Nitride. Science 2014, 343 (6175), 1125-1129. https://doi.org/10.1126/science.1246833.

(5) Phillips, C.; Gilburd, L.; Xu, X. G.; Walker, G. C. Surface and Volume Phonon Polaritons in Boron Nitride Nanotubes. J. Phys. Chem. Lett. 2019, 10 (17), 4851-4856. https://doi.org/10.1021/acs.jpclett.9b01829.

(6) Hertel, T.; Walkup, R. E.; Avouris, P. Deformation of Carbon Nanotubes by Surface van Der Waals Forces. Phys. Rev. B 1998, 58 (20), 13870-13873. https://doi.org/10.1103/PhysRevB.58.13870. 